\title{
Um Sistema Adaptativo e Colaborativo para Minimizar Congestionamentos utilizando Comunicação entre Veículos
}

\author{
Thiago S. Gomides ${ }^{1,2}$, Robson E. De Grande ${ }^{2}$ \\ Fernanda S.H. Souza ${ }^{1}$ e Daniel L. Guidoni ${ }^{1}$ \\ ${ }^{1}$ Departamento de Ciência da Computação \\ Universidade Federal de São João Del Rei, (UFSJ) - MG - Brasil \\ ${ }^{2}$ Department of Computer Science - Brock University \\ St. Catharines, Ontario, Canada. \\ \{gomides,f.sumika, guidoni\}@ufsj.edu.br, rdegrande@brocku.ca
}

\begin{abstract}
Vehicular Traffic Management become an important challenge for large cities due to the constant growth of vehicles. As the road mesh does not increase as well as the number of vehicles in the streets, technological solutions for the traffic congestion rise as alternative and easy-to-use applications. This work presents an adaptive and Distributed Traffic Management System for VANETS. The proposed solution is based on V2V communication and the local view of traffic congestion. During its displacement in a road, the vehicle monitors its traveled distance and the expected one considering a free-flow traffic condition. This measurement is used to classify the traffic congestion. The proposed System uses a proactive and reactive dissemination protocols to create a distributed database of traffic congestion among vehicles. Using only local information, vehicles verifies alternatives routes with lower traffic congestion. The proposed solution is compared with three literature solutions, named DIVERT, PANDORA and SGTD. Our results showed that our proposal presents better results regarding network and traffic congestion metrics.
\end{abstract}

Resumo. O gerenciamento de tráfego de veículos se tornou um desafio importante para as grandes cidades devido ao constante crescimento de veículos. Como a malha viária não aumenta como o número de veículos nas ruas, soluções tecnológicas para o gerenciamento do tráfego surgem como aplicações alternativas e fáceis de usar. Este trabalho apresenta um sistema de gerenciamento de tráfego adaptável e distribuído para VANETS. A solução proposta é baseada na comunicação V2V e na visão local do congestionamento do tráfego. Durante o deslocamento em uma rua, o veículo monitora a distância percorrida e a esperada considerando condições de tráfego em fluxo livre. Essa medida é usada para classificar o congestionamento do tráfego. O sistema proposto utiliza protocolos de disseminação proativa e reativa para criar um banco de dados distribuído contendo informações sobre congestionamento de tráfego entre veículos. Usando apenas informações locais, os veículos verificam rotas alternativas com menor congestionamento de tráfego. A solução proposta é comparada com três soluções da literatura, denominadas DIVERT, PANDORA e SGTD. Nossos resultados mostraram que nossa proposta apresenta melhores resultados em relação às métricas de congestionamento de rede e tráfego veicular. 


\section{Introdução}

Com o agrupamento populacional nos centros urbanos, a gestão da mobilidade urbana é um importante e recorrente desafio nas grandes cidades. A Organização das Nações Unidas (ONU) classifica esse desafio como um dos 17 Goals to Transform Our World que consiste em, até 2030, tornar as cidades mais inclusivas, seguras, resilientes e sustentáveis. No entanto, a falta de investimento na infraestrutura, distancia os centros urbanos dessas condições ideais. Segundo o estudo The Future of Urban Mobility 2.0 [Van Audenhove et al. 2014], estima-se que no ano de 2014, 53\% da população mundial estava concentrada nos grandes centros urbanos. Além disso, para 2050 é esperado um crescimento de $14 \%$ e essa concentração é atribuída, principalmente, em razão das atividades essenciais como o trabalho, a educação e a busca por melhores oportunidades e qualidade de vida [Mabogunje 1970]. A falta de planejamento urbano acentua os problemas da mobilidade, fazendo com que mais pessoas utilizem meios de transporte privados, contribuindo para o surgimento de congestionamentos. Em termos econômicos, estima-se que o custo do trânsito na Europa, até 2025, ultrapassará os 183 bilhões de Euros [Cookson and Pishue 2016]. Além disso, os congestionamentos impactam a qualidade de vida das pessoas [Cookson and Pishue 2016], tornando as cidades ambientes mais estressantes e menos produtivos [Ekblad 1993].

Para a redução desses impactos, popularizam-se as soluções tecnológicas, de menor custo de implantação e influência na vida das pessoas. Assim, Sistemas de Gerenciamento de Tráfego Veiculares são soluções de tecnologia da informação e comunicação que consideram características das vias e dos usuários que, dessa forma, proporcionam mecanismos capazes de melhorar a utilização da infraestrutura urbana disponível. Em geral, esses sistemas avaliam as condições dos deslocamentos dos veículos para o monitoramento do tráfego, a detecção dos congestionamentos e a sugestão de novas rotas com o objetivo de minimizar congestionamentos [Shahgholian and Gharavian 2018]. No desenvolvimento dos sistemas de gerenciamento de congestionamentos, destaca-se a utilização das Redes Veiculares (Vehicular Ad-hoc NETtorks - VANETs) como um importante componente no projeto das soluções.

A utilização das redes veiculares contribuem nesse processo com a incorporação de tecnologias de sensoriamento, interfaces de comunicação sem fio e capacidade de armazenamento e processamento nos veículos [Ahmed and Gharavi 2018]. Dessa forma, os veículos com capacidade de comunicação e sensoriamento são capazes de monitorar e compartilhar as condições do seu deslocamento considerando os problemas da mobilidade urbana, como: a redução dos congestionamentos e dos acidentes, redução da emissão de gases poluentes, a diminuição do tempo de viagem dos veículos e o aumento da vazão do fluxo das vias. A utilização de VANETs no projeto de soluções para minimizar congestionamentos em centros urbanos vem sendo pesquisada pelos autores em vários trabalhos da literatura, tais como [Wang et al. 2014], [de Souza et al. 2017], [Lourenço et al. 2018], [Brennand et al. 2017] e [Pan et al. 2017].

Este trabalho propõe um sistema de gerenciamento de tráfego veicular utilizando protocolos de disseminação de dados entre veículos para a transmissão de informações sobre tráfego de veículos em centros urbanos. O sistema proposto é baseado na observação local do veículo para estimar as condições de tráfego. Sempre que um veículo verificar a existência de crescimento do tráfego/congestionamento durante sua rota, eles devem di- 
vulgar estas informações utilizando o protocolo de disseminação proativa e adaptativa de informações. Considerando as informações de tráfego recebidas e, quando necessário, o veículo verifica rotas alternativas com menor congestionamento. Nos casos de ausência de informações, o veículo executará o protocolo reativo de descoberta de informações de tráfego. O sistema proposto foi comparado às soluções da literatura, apresentando melhores resultados relacionados às métricas de eficiência de rede e tráfego de veículos.

O restante deste trabalho está organizado da seguinte maneira. A seção 2 apresenta as soluções da literatura para sistemas de gerenciamento de tráfego. A Visão Geral do sistema proposto e seus princípios de projeto são apresentados na Seção 3. Os protocolos de disseminação proativa e reativa são descritos na Seção 4. A Seção 5 mostra a análise de desempenho. Conclusões e trabalhos futuros são apresentados na Seção 6.

\section{Trabalhos Relacionados}

Considerando a infraestrutura de comunicação, os sistemas de gerenciamento de tráfego veicular podem ser divididos em três abordagens: (i) centralizado, (ii) descentralizado e (iii) distribuído. Nas soluções centralizadas, os veículos recebem rotas alternativas com menor congestionamento de um servidor central que possui uma visão global das informações de tráfego. Essas soluções exigem um alto custo de comunicação [Pan et al. 2017, Wang et al. 2015]. Sistemas de navegação veicular (VNS), como Google Maps, INRIX, Waze e Apple Maps também são exemplos de soluções centralizadas. Por outro lado, em soluções descentralizadas, as Road-Side Units (RSUs) são implantadas nas ruas para receber, processar e disseminar informações de tráfego e rotas alternativas. No entanto, o uso da RSU introduz um custo extra para o gerenciamento de tráfego [Lourenço et al. 2018, Brennand et al. 2017]. Finalmente, as soluções distribuídas são baseadas apenas na comunicação entre veículos. Os veículos, enquanto se deslocam, disseminam e recebem informações de trânsito para estimar localmente rotas alternativas [de Souza et al. 2017].

Em [Pan et al. 2012], os autores apresentam uma solução chamada Random kShortest Path ( $k \mathrm{SP})$, que consiste em um algoritmo de gerenciamento de tráfego. Para cada veículo, $k$-caminhos alternativos são calculados para obter um fluxo distribuído de veículos e, ao receber os caminhos, o veículo escolhe um deles aleatoriamente. Além disso, a diferença entre as $k$-rotas mais rápidas e as $k$-rotas mais lentas não devem ser superiores a $20 \%$ para evitar um aumento significativo no tempo de viagem. Os autores desenvolveram o R $k$ SP para apresentar o DIVERT: um sistema de gerenciamento de tráfego de veículos para reduzir congestionamentos [Pan et al. 2017]. DIVERT é um sistema centralizado que usa o tempo de viagem de cada segmento de estrada para calcular novos rotas. Periodicamente, o servidor central solicita o tempo de viagem de cada segmento de estrada para veículos. O servidor analisa o fluxo de tráfego global e encaminha novas rotas de menor custo para os veículos. A coleta de dados pelo servidor central é feita utilizando comunicação 4G.

Um Sistema de Gerenciamento de Tráfego Distribuído para Redes Veiculares (SGTD) é proposto em [Gomides et al. 2019]. A partir de análises momentâneas dos deslocamentos, o sistema permite que os veículos sejam capazes de detectar e classificar os congestionamentos nos centros urbanos. Para isso, as informações coletadas durante as viagens são utilizadas pelos veículos para a decisão de novas rotas. Cada veículo, classi- 
fica o congestionamento considerando a relação entre a distância percorrida e a distância esperada em condições de fluxo livre. Sempre que alterações no fluxo são detectadas, os veículos devem disseminar estas informações para a primeira vizinhança, ou seja, para que a decisão de próxima conversão seja tomada. Ao se aproximar das esquinas, cada veículo escolhe o próximo segmento a ser percorrido e esse processo é repetido durante todo o deslocamento.

Em [de Souza et al. 2017], os autores apresentam o Preventing trAffic congestioN through a fully-Distributed Rerouting Algorithm - PANDORA, um sistema para o controle de tráfego totalmente distribuído que considera a disseminação oportunista do conhecimento. Usando a comunicação entre veículos, o PANDORA utiliza um modelo de conteúdo flutuante e informações de tráfego localizadas geograficamente em áreas críticas. A área crítica define a região onde o sistema deve ser executado para diminuir o congestionamento do tráfego. A área crítica é dividida em sub-regiões e os veículos que se deslocam em uma sub-região específica recebem o tempo de viagem de todas as ruas dentro dela. Usando um intervalo de beacon de 2 segundos, os veículos compartilham suas localizações e velocidades geográficas com todos os veículos dentro da sub-região para classificar o deslocamento do veículo. Considerando as informações recebidas e com base no Nível de Serviço (LOS) proposto no Manual de Capacidade Rodoviária (HCM) [HCM 2010], os veículos estimam rotas alternativas com menor tempo de viagem. Um parâmetro importante do PANDORA é a definição da área crítica, uma vez que afeta o número de mensagens transmitidas para executar a solução de gerenciamento de tráfego veicular.

\section{Sistema de Gerenciamento de Tráfego Veicular Proposto}

Esta seção apresenta todos os conceitos e decisões de projeto para a implementação do sistema de gerenciamento de tráfego veicular utilizando apenas o modelo de comunicação Veículo-para-Veículo - V2V. Serão apresentados também os protocolos de disseminação de dados que combinam diferentes comportamentos na disseminação de informações sobre congestionamentos. A disseminação de informação é realizada a partir de um aspecto multi-paradigma descrito em duas formas: proativa e reativa. Na forma proativa, os veículos disseminam as informações quando alterações no fluxo de veículos são detectadas e, na forma reativa, os veículos podem requisitar essas informações. O sistema de gerenciamento de tráfego proposto aborda as seguintes questões: (i) avaliação do congestionamento nas ruas/rotas onde os veículos realizam o percurso; (ii) compartilhamento colaborativo e adaptativo da percepção local do tráfego veicular; e (iii) estimativa de rotas alternativas usando informações recebidas de outros veículos.

A Figura 1(a) apresenta a visão geral do sistema proposto. Os veículos, a partir da comunicação V2V, compartilham sua observação local do tráfego com os veículos de sua vizinhança. Ao receber uma nova mensagem de informações de trânsito, o veículo azul pode encontrar um caminho alternativo sem congestionamentos ou com um congestionamento menor (representado pela linha verde). Em nosso trabalho, a percepção local do tráfego é verificada considerando o deslocamento do veículo (distância percorrida) proposto em [Gomides et al. 2019]. De maneira geral, o contexto do sistema de gerenciamento de tráfego pode ser definido da seguinte forma: 


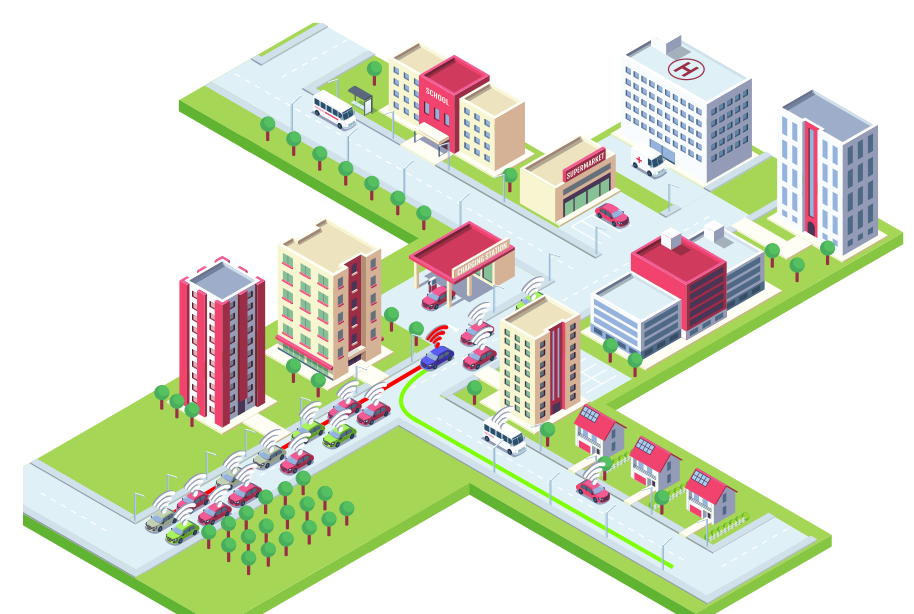

(a) ON-DEMAND modelo de alto nível

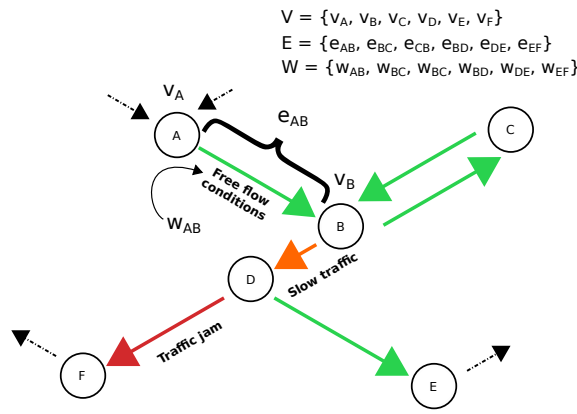

(b) Grafo $\mathrm{G}=(\mathrm{V}, \mathrm{E})$ representa o mapa de ruas da cidade da Figura 1(a).

Figura 1. Visão geral do sistema

Definição 1 (Ambiente veicular). Considere o ambiente de veicular definido por um grafo direcionado e ponderado $G=(V, E)$, onde $V=\left\{v_{i}, i=0 \ldots n\right\}$ é o conjunto de vértices e $E=\left\{\left(v_{i}, v_{j}\right) \leftrightarrow v_{i} \in V \wedge v_{j} \in V\right\}$ é o conjunto de arestas onde $\left(v_{i}, v_{j}\right) \neq\left(v_{j}, v_{i}\right)$. O conjunto de arestas representa os segmentos da rua (rua entre dois cruzamentos) e o conjunto de vértices representa os cruzamentos do mapa da cidade. Cada aresta $e_{i j}=\left(v_{i}, v_{j}\right)$ representa o segmento de rua $e_{i j}$ conectando a interseção $i$ e j com um custo associado $w_{i j}$ que representa o custo de atravessá-lo. O conjunto de ponderação pode ser representado por $W=\left\{w_{i j}, i \neq j\right\}$ onde $i$ e $j$ são iguais à cardinalidade de $E$ e $w_{i j} \rightarrow R_{+}^{*}$. Seja $N$ o conjunto de veículos (nós) onde $N=\left\{n_{k}, k=0 \ldots m\right\}$ e $m$ o número de veículos no ambiente.

Nesse ambiente, cada veículo é capaz de processar, analisar, comunicar e tomar decisões distribuídas. Para isso, todos os veículos são equipados com: Unidade de bordo (OBU), interface de comunicação IEEE 802.11 p, sensores, receptor do sistema de posição global (GPS) e o mapa com as ruas e características da cidade. O mapa das ruas da cidade é representado pelo grafo direcionado e ponderado $G=(V, E)$, ilustrado na Figura 1(b). Além de conter informações sobre condições de tráfego (as cores vermelho e verde representam congestionamentos e condições de fluxo livre, respectivamente), o grafo $G$ também possui atributos como dimensões das ruas, número de faixas, posições de semáforos, velocidade máxima permitida etc.

Durante o percurso em cada rua pertencente ao seu trajeto da origem ao destino, os veículos classificam as alterações das condições do tráfego realizando a associação entre a Distância Percorrida pelo veículo e a Distância Esperada que o veículo deveria percorrer em fluxo livre. A diferença entre as duas estimativas de distancias define um fator e congestionamento, onde veículos que possuem a distância percorrida similar a distância esperada em fluxo livre possuem o fator de congestionamento de 1 . Se essa diferença for maior do que $90 \%$ o fator de congestionamento é definido como 10, indicando uma rua totalmente congestionada, uma vez que o veículo realizou apenas um pequeno percurso nela. $\mathrm{O}$ fator de congestionamento é um número inteiro de 1 a 10 e sempre que o veículo perceber alterações de valor, o protocolo de Disseminação proativa de dados 

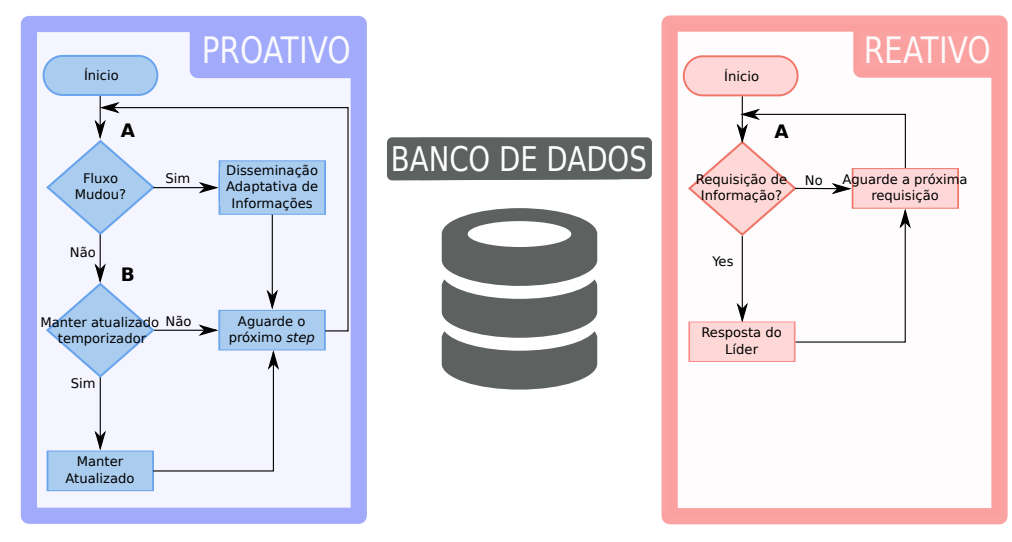

Figura 2. Funcionamento geral dos protocolos de disseminação

é executado para o envio da mensagem contendo o fator estimado para veículos vizinhos.

Cada veículo possui uma base de dados local para armazenar as informações recebidas e, ao aproximar das interseções, eles verificam se existem rotas alternativas com menos congestionamento. Se o veículo verificar que ele não possui informações de tráfego de alguma rua adjacente da interseção em análise, ele executa o protocolo de Disseminação reativa de dados para descobrir o fator de congestionamento da rua em questão. Para o cálculo de uma nova rota, cada veículo deve atualizar $w_{i j} \forall e_{i j} \in G(V, E)$ utilizando as informações do seu banco de dados e considerando $w_{i j}=$ FatorCongestionamento $_{i j} \times T E_{i j}$, onde $T E_{i j}$ representa o tempo de percurso esperado da rua $e_{i j}$ em condições de fluxo livre. Rotas alternativas são encontradas aplicando um algoritmo de caminho de custo mínimo em $G(V, E)$.

\section{Protocolos de Disseminação de Dados}

Os veículos monitoram as condições de seus deslocamentos para a execução adequada dos protocolos de disseminação. A Figura 2 apresenta a visão geral dos protocolos propostos e seus comportamentos.

\subsection{Disseminação Proativa de Informações}

O protocolo de Disseminação Proativa de Informações é responsável por fornecer e compartilhar informações suficientes para decisões de rotas alternativas de menor custo, ou seja, com menor interferência dos congestionamentos. Este protocolo foi projetado para ser executado em um ambiente distribuído e altamente móvel usando a comunicação V2V. Com base nas informações estimadas de congestionamento, a Disseminação Proativa possui dois comportamentos: (i) quando há alteração no fluxo e (ii) quando há necessidade de manter informações de tráfego. No primeiro, cada veículo que detecta um aumento no fluxo deve disseminar proativamente essas informações de acordo com o modelo de compartilhamento de informação adaptativo (Figura 2, Rótulo A) e o veículo aguarda a próxima etapa para realizar uma nova análise de deslocamento. Se o fluxo de veículos não possui alterações, o veículo verificará a necessidade de manter estas informações 


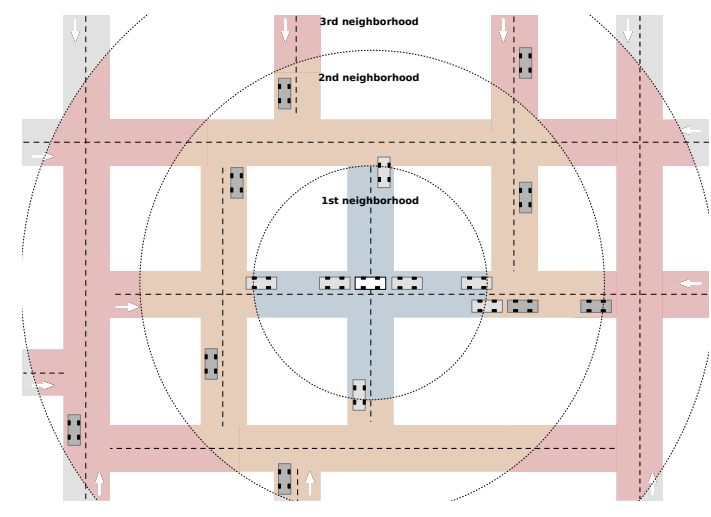

(a) Alcance de múltiplas vizinhanças

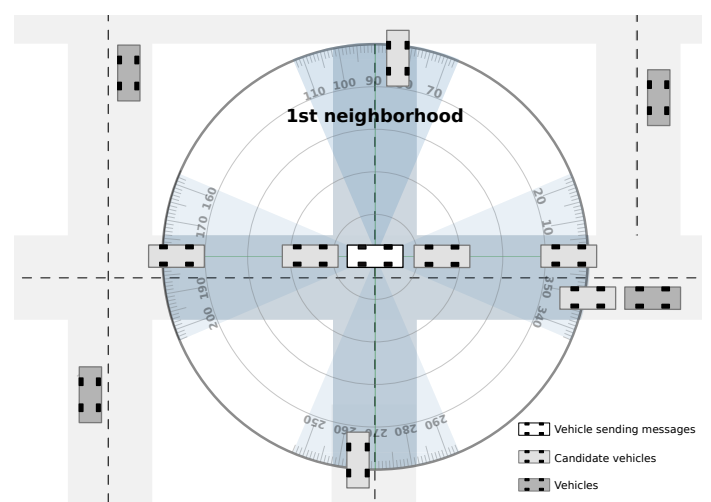

(b) Destaque a primeira vizinhança

Figura 3. Disseminação Adaptativa de Informações

atualizadas, ou seja, o veículo verificará o cronômetro de Manter Atualizado (Figura 2, Rótulo B). Se o cronômetro estiver finalizado, o veículo realiza a disseminação das informações sobre a condição do tráfego, caso contrário, ele aguardará a próxima etapa. Esses comportamentos são detalhados a seguir.

A disseminação adaptativa de informação é executada com base na mudança do fator de congestionamento e sua intensidade. Sempre que modificações no fluxo veicular são detectadas, os veículos devem avaliar a necessidade de disseminar esta informação à sua vizinhança. Para isso, cada veículo consulta o seu banco de dados local em busca de informações do tráfego, que contêm as informações recebidas e estimadas contidas em tuplas $<r u a_{i d}$, congestionamento ${ }_{\text {rua }}>$. Quando o veículo está movendo-se na $r u a_{i d}$ e o fluxo veicular é alterado, ele verifica se o banco de dados contém informações sobre $r u a_{i d}$ e, no caso de falha na verificação, o veículo atualiza seu banco de dados e dissemina a informação estimada com seus vizinhos. Esse caso ocorre quando o veículo ainda não recebeu/estimou nenhuma informação sobre o $r u a_{i d}$. Se o banco de dados contiver informações sobre a rua em questão, o veículo compara as duas estimativas e se a recebida/estimada for maior, o veículo atualiza o banco de dados e dissemina a estimativa. Este cenário indica que o fluxo de tráfego está aumentando na $r u a_{i d}$ e os veículos vizinhos devem ser notificados. No entanto, se o fluxo for menor comparado ao do banco de dados, o compartilhamento de informação é suprimido. O banco de dados é atualizado com o fluxo estimado mais antigo entre os dois, onde consideramos que o veículo que percorreu uma distância maior na estrada possui um melhor conhecimento do fluxo de tráfego.

Os veículos executam esse procedimento de maneira adaptativa, ou seja, a área de abrangência da disseminação das informações dependerá da intensidade de tráfego. Se o veículo estima um fator de congestionamento de [1 ou 2], as informações são disseminadas utilizando um $T T L=1$, ou seja, o tempo de vida de um salto. Por outro lado, com o crescimento dos congestionamentos, as informações devem atingir maiores distâncias para auxiliar os veículos a encontrar rotas alternativas, uma vez que o congestionamento pode estar ocorrendo em uma região e não apenas em uma rua. Para isso, quando o fator de congestionamento for [3 ou 4] $\rightarrow T T L=2$, [5,6 ou 7] $\rightarrow T T L=3$ e [8,9 ou 10] $\rightarrow T T L=4$. Verificamos que não há necessidade do compartilhamento de informações para uma vizinhança maior do que 4 saltos de distância, ou seja, os veículos não 
são capazes de encontrar melhores rotas. Um outro fator considerado é a quantidade de mensagens, que é reduzida ao limitar a área de disseminação.

O cenário urbano ilustrado na Figura 3(a) mostra diferentes áreas da disseminação adaptativa de informações. Considerando o veículo de cor branca posicionado no centro da figura, $T T L=1$ ilustra a primeira vizinhança, $T T L=2$ ilustra a segunda vizinhança e etc. Para valores de $T T L \geq 2$, é necessário criar um mecanismo de retransmissão de mensagens para reduzir o problema de Broadcast storm. Para isso, definimos a região de destino como coordenadas angulares centralizadas no veículo emissor, ou seja, o veículo que está compartilhando as mudanças de seu fluxo. Este processo é ilustrado na Figura 3(b). Somente veículos dentro dos intervalos angulares podem encaminhar a mensagem e a área de encaminhamento é dividida considerando o mapa da cidade e o número de ruas adjacentes. As regiões angulares mudam com base no layout das ruas e isso se deve ao fato de os veículos se deslocarem apenas dentro das vias urbanas. Para cada rua adjacente, consideramos uma região angular de $45^{\circ}$ onde a disseminação de dados pode ser executada. Para controlar melhor o problema de transmissão, apenas um veículo dentro de cada intervalo angular encaminha a mensagem. Para isso, o veículo que tem uma distância maior do remetente encaminha a mensagem primeiro, cancelando as outras transmissões. É importante ressaltar que uma mensagem de um veículo dentro de um intervalo angular não cancela o procedimento de encaminhamento de outro veículo posicionado em outro intervalo angular. Dessa forma, a sobrecarga do acesso ao meio sem fio é minimizada, pois apenas um veículo de cada intervalo angular retransmite a mensagem, reduzindo o número de mensagens duplicadas. Esse processo ocorre no caso de várias vizinhanças $(T T L \geq 2)$ com o objetivo de fornecer informações de tráfego eficientes e suficientes para um maior número de veículos na região congestionada.

O objetivo desse procedimento descrito é minimizar o número de mensagens para criar um conhecimento distribuído sobre as condições de tráfego das ruas. No entanto, um equilíbrio entre o controle de contenção da informação de congestionamento e o tempo de vida das informações distribuídas deve ser considerado, uma vez que os veículos continuarão sua rota, mas novos veículos passarão pela rua e precisarão de informações sobre congestionamento. Para impedir que as informações de tráfego sejam extintas, utilizamos um mecanismo de Manter Atualizado. Nesse caso, a disseminação da informação é realizada somente na primeira vizinhança, para reduzir a sobrecarga do meio sem fio e o número de mensagens transmitidas. Quando o veículo começa a se mover em uma nova rua, um temporizador de manutenção da informação é iniciado a cada $\mathbf{T}$ unidades de tempo. Se o veículo receber uma informação compartilhada por outro veículo ou quando o veículo detectar um crescimento do fator de congestionamento, o temporizador é reiniciado, pois não há ausência de informações. Quando o temporizador finaliza, o veículo verifica se possui as informações da rua em seu banco de dados e, se sim, as informações são disseminadas para seus vizinhos. Após, o temporizador é reiniciado para manter atualizadas as informações da rua. É importante enfatizar que a manutenção do conhecimento é realizada apenas quando não há alteração nos níveis de congestionamento compartilhados pelo veículo e após $\mathbf{T}$ unidades de tempo.

\subsection{Disseminação Reativa de Informações}

O protocolo de disseminação reativa é responsável por permitir que os veículos solicitem informações quando ocorrerem falhas no processo proativo de disseminação de dados. 
Essas falhas podem ser causadas por vários fatores, como: colisões de pacotes devido à sobrecarga da rede (diferentes aplicações podem ser executadas usando a interface de comunicação sem fio), redes esparsas ou desconectadas, atenuação de sinal entre outros. Sempre que necessário, os veículos podem solicitar informações de congestionamento para veículos em ruas adjacentes.

A solicitação reativa de informações é dividida em três etapas: (i) enviar mensagem de solicitação, (ii) realizar eleição de líder e (iii) responder para o veículo solicitante o fator de contenção da rua. Na primeira etapa, o veículo realiza a solicitação ao detectar a ausência de informações sobre ruas adjacentes (Figura 2, Rótulo C). Para isso, cada veículo envia uma mensagem de requisição para os veículos dentro da rua específica, ou seja, rua sem informações de trânsito. Os veículos que receberam a mensagem de requisição executam a eleição de um líder, onde o líder será responsável em responder a requisição (Figura 2, Rótulo D). Ao receber a requisição, cada veículo se auto elege líder agendando o envio de uma mensagem de líder. $\mathrm{O}$ agendamento é realizado de maneira inversamente proporcional ao tempo de percurso na via, onde o veículo com o maior tempo de percurso enviará a mensagem de líder primeiro. Dessa forma, o líder é o veículo que possui maior experiencia do fluxo dentro do raio de comunicação do veículo solicitante. Ao receber uma mensagem de líder, os demais veículos cancelam os seus respectivos agendamentos.

Na terceira etapa, o líder cria uma mensagem de resposta para o veículo solicitante contendo o fator de congestionamento presente em sua base de dados local. Por outro lado, se a partir de uma eleição de líder anterior a rua possuir um líder, o líder responderá a requisição imediatamente, cancelando uma eventual nova eleição de líder. Os veículos e suas respectivas percepções de tráfego podem mudar com o passar do tempo e, com a presença de um líder, a resposta pode ser realizada de maneira imediata. É importante ressaltar que o tempo de vida de um líder eleito é sua permanência na rua. Assim, quando o líder mudar de rua, uma nova eleição acontecerá, uma vez que os veículos irão agendar uma eleição de líder e nenhuma resposta imediata será observada. É importante ressaltar também que a mensagem de resposta à requisição poderá ser utilizada por outros veículos que a receberem além do veículo requisitante.

\section{Análise de Desempenho}

O desempenho da solução proposta é avaliado com simulações da comunicação e mobilidade de um ambiente veicular considerando um cenário com características e parâmetros do mundo real. Para isso, foi utilizado o SUMO 0.25 (Simulation of Urban MObility) [Lopez et al. 2018] para criar e gerenciar a mobilidade de veículos, OMNET++ 5.1.1 [Varga and Hornig 2008] e Veins 4.6 [Sommer et al. 2011] como framework de comunicação veicular usando o padrão 802.11 p. As simulações foram realizadas considerando um recorte da região metropolitana de Portland - EUA, obtido através da ferramenta OpenStreetMap [OpenStreetMap contributors 2017], possuindo uma região de 17 $\mathrm{km}^{2}$ conforme ilustrado na Figura 4. O OpenStreetMap fornece ao mapa as dimensões das ruas, direções, semáforos, limites de velocidade, preferência de veículo, entre outros.

A solução proposta foi avaliada em comparação com as soluções PANDORA, SGTD, DIVERT e OVMT (Original Vehicle Mobility Trace), onde as rotas dos veículos não são alteradas. As rotas iniciais dos veículos são definidas de maneira aleatória (origem 


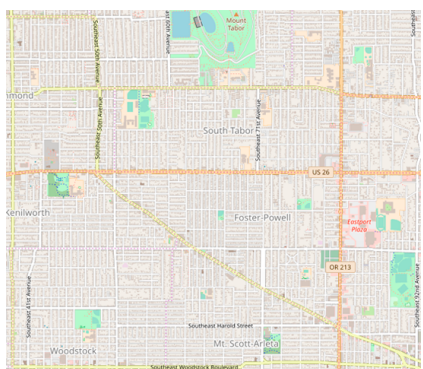

(a) OpenStreetMap

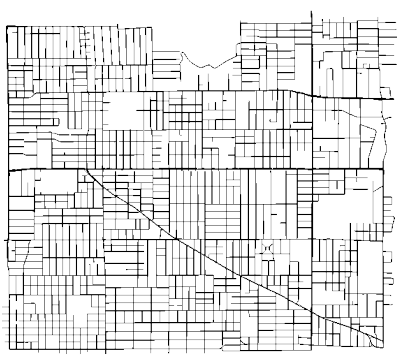

(b) SUMO

Figura 4. Mapa da cidade de Portland, Estados Unidos

e destino) e o percurso é realizado pelo menor caminho. Foram realizadas 33 simulações com diferentes traces de mobilidade para obtermos um intervalo de confiança de $95 \%$. $\mathrm{O}$ raio de comunicação dos veículos é de $250 \mathrm{~m}$. Na solução proposta, o cronometro "Manter Atualizado" é iniciado a cada 10 segundos. A área crítica do PANDORA é toda a região de $17 \mathrm{~km}^{2}$. As soluções foram avaliadas considerando o impacto da rede de comunicação e a eficiência do tráfego. Para a avaliação da comunicação, consideramos duas métricas: Mensagens transmitidas e Colisão de pacotes. A primeira métrica representa o número de mensagens transmitidas para a execução do sistema de gerenciamento de tráfego considerando as soluções distribuídas, enquanto a segunda avalia a colisão média de pacotes devido à sobrecarga da rede.

A eficiência do tráfego foi avaliada considerando as métricas Tempo de Viagem e Tempo perdido. O tempo de viagem é o tempo médio para que todos os veículos possam concluir suas viagens. O tempo perdido em trânsito apresenta a diferença entre o tempo gasto no cenário atual com as interrupções de fluxo em razão dos congestionamentos de trânsito em comparação ao cenário de condições de fluxo livre sem congestionamentos. Valores mais altos dessas métricas significam mais interrupções no tráfego e, consequentemente, mais custos com tempo, combustível e poluição ambiental. Valores mais baixos significam que o sistema pode reduzir os impactos dos engarrafamentos. Os resultados foram obtidos variando a densidade de veículos no cenário, onde 50 veículos $/ \mathrm{km}^{2}$ representa um cenário sem congestionamento e 350 veículos $/ \mathrm{km}^{2}$ representa um cenário com muitos congestionamentos. Além disso, também avaliamos o desempenho a partir de uma função de distribuição cumulativa (CDF), que apresenta a avaliação entre os tempos relativos de viagem comparando as soluções de gerenciamento de tráfego com OVMT.

\subsection{Avaliação de Rede}

O principal objetivo desta avaliação é analisar o custo da comunicação para executar as soluções distribuídas que usam o padrão de comunicação IEEE 802.11p e que consideram a disseminação de dados em múltiplas vizinhanças. As soluções centralizadas usam comunicação com RSU ou com a infraestrutura celular para executar o gerenciamento de tráfego. A solução SGTD realiza a disseminação de informações considerando somente a primeira vizinhança. A Figura 5 apresenta os resultados para mensagens transmitidas e colisões de pacotes considerando diferentes densidades de veículos no cenário. A solução PANDORA compartilha as informações de tráfego usando um intervalo de 2 segundos para as mensagens do tipo beacons, o que leva a um alto número de mensagens transmitidas (Figura 5(a)). O sistema proposto compartilha as informações de tráfego em 


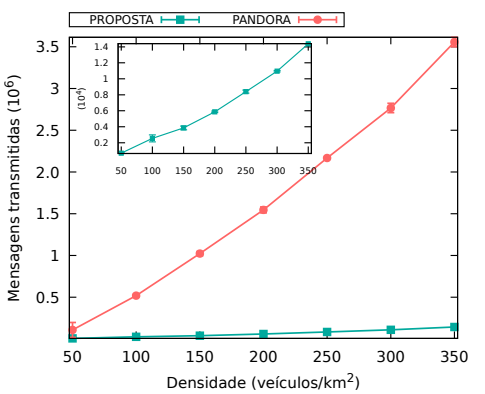

(a) Mensagens transmitidas

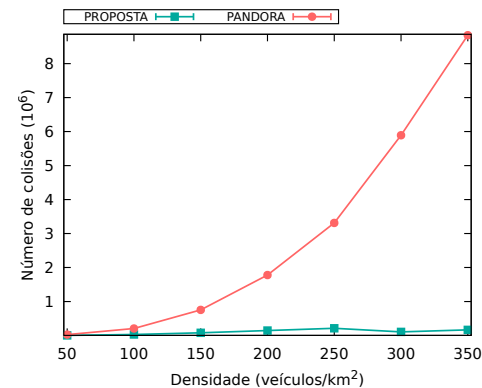

(b) Colisão de Pacotes

Figura 5. Métricas de Redes

situações específicas: (i) quando existem alterações no fluxo, (ii) quando o cronômetro "Manter Atualizado"finaliza ou (iii) a partir da solicitação de informações. Dessa forma, o sistema consegue reduzir a quantidade de mensagens transmitidas. Em média, a solução proposta transmite $10^{2}$ vezes menos mensagens em comparação com o PANDORA para as diferentes quantidades de veículos no cenário.

A Figura 5(b) mostra o número de colisões de pacotes para executar as duas soluções. À medida que a PANDORA transmite mais mensagens, ele sobrecarrega o acesso ao meio sem fio, causando um alto número de colisões de pacotes. É possível verificar que mesmo transmitindo um número menor de mensagens, o número de colisões de pacotes do sistema proposto é diferente de zero. Nesse caso, a disseminação adaptativa de informação ou a manutenção das informações de tráfego podem não funcionar adequadamente, mostrando a necessidade do protocolo de disseminação reativa de informações.

\subsection{Avaliação de Tráfego}

A Figura 6 mostra os resultados referentes as métricas de tráfego veicular e o tempo de viagem é ilustrado na Figura 6(a). Para as menores densidades de veículos (50 ou 100 veículos $/ \mathrm{km}^{2}$ ), ou seja, com poucos pontos de congestionamento, todas as soluções têm resultados semelhantes. O crescimento do número de veículos contribui para o surgimento dos congestionamentos e a solução proposta e o DIVERT apresentam melhores resultados em comparação com as outras soluções. É importante destacar que os resultados apresentados pela solução PANDORA nas densidades avaliadas apresentam tempos de viagens semelhante ao OVMT. Isso se deve ao fato de o PANDORA apresentar melhores resultados somente quando uma pequena área crítica do mapa apresenta congestionamento [de Souza et al. 2017]. Quando a densidade de veículos é de 300 veículos $/ \mathrm{km}^{2}$, o sistema proposto é capaz de reduzir o tempo de viagem em $60 \%, 58 \%$, 42\% e $14 \%$ em comparação com o OVMT, PANDORA, SGTD e DIVERT, respectivamente. Assim, destaca-se que a utilização da distância percorrida em comparação com a distância esperada em fluxo livre apresenta melhores resultados em comparação ao HCM utilizado pelo PANDORA ou ao número de veículos utilizados pelo DIVERT. A Figura 6(b) mostra o tempo perdido nos engarrafamentos. À medida que o cenário possui congestionamentos, as soluções de gerenciamento de tráfego conseguem encontrar rotas alternativas com menor congestionamento, reduzindo o tempo perdido pelos veículos para realizar o trajeto. Essa redução afeta diretamente o consumo de combustível e as emissões de $\mathrm{CO}_{2}$, que também são consideradas questões a serem observadas no projeto de soluções para o 


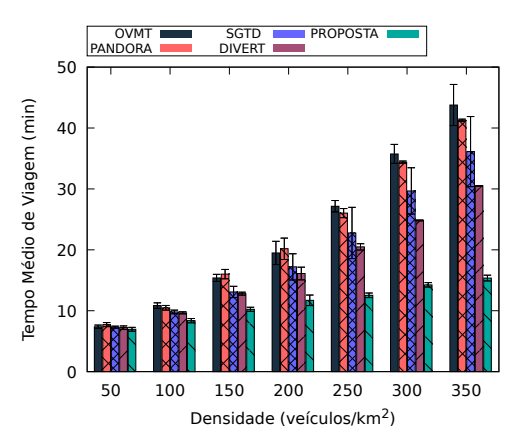

(a) Tempo de viagem

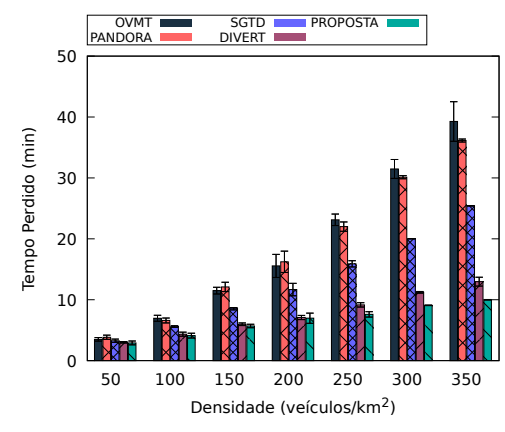

(b) Tempo perdido

Figura 6. Métricas de Tráfego Veicular

gerenciamento de tráfego veicular.

A Figura 7 avalia a relação entre as soluções para minimizar congestionamentos e o OVMT considerando uma CDF do tempo de viagem e tempo perdido em diferentes densidades de veículos na rede. $\mathrm{O}$ eixo x representa a razão entre as soluções e o eixo y representa a porcentagem acumulada de veículos que satisfazem a razão. A Figura 7(a) mostra o tempo de viagem relativo considerando uma densidade de tráfego normal (100 veículos $/ \mathrm{km}^{2}$ ). O DIVERT, que tem uma visão global do congestionamento do tráfego, organiza o fluxo de tráfego de veículos para diminuir o tempo de viagem. É possível verificar se $70 \%$ dos veículos que executam o DIVERT têm um tempo de viagem inferior a $0,5 \times$ OVMT (tempo de viagem relativo $\leq 0.5$ ). O PANDORA, DIVERT e a solução proposta têm $80 \%$ dos veículos com um tempo de viagem menor que o OVMT (tempo de viagem relativo 1), onde o SGTD possui apenas $70 \%$ dos veículos. É importante ressaltar que as soluções não são capazes de promover a diminuição no tempo de viagem de todos os veículos. A estimativa de rotas alternativas pode apresentar congestionamentos que não foram estimados previamente, ou seja, veículos alteram suas rotas para os mesmos trajetos, resultando em novos congestionamentos. Um outro fator é que os veículos podem alterar suas rotas, mas o congestionamento do trajeto anterior estava a tempo de diminuir, ou seja, uma "má decisão".

A Figura 7(b) mostra o tempo perdido de todas as soluções. O DIVERT, SGTD, solução proposta e PANDORA reduzem o tempo perdido em 90\%, 60\%, 77\% e 76\%, respectivamente. Quando a densidade de veículos é de 350 veículos $/ \mathrm{km}^{2}$ (Figuras 7(c) e 7(d)), pode-se perceber que a solução proposta possui os melhores resultados em comparação com as demais soluções. Dessa forma, a avaliação do tráfego em conjunto com os protocolos de disseminação proativa e reativa de informações consegue reduzir o tempo de viagem e tempo perdido em cenários com muitos congestionamentos.

\section{Conclusões}

Este trabalho propõe um sistema de gerenciamento de tráfego adaptável e distribuído para redes veiculares. $\mathrm{O}$ sistema proposto utiliza apenas comunicação entre veículos para criar uma base de dados local contendo informações de tráfego. O principal objetivo do sistema proposto é diminuir o tempo médio de viagem de veículos com baixo impacto na quantidade de mensagens transmitidas em cenários urbanos e congestionados. O sistema proposto realiza a disseminação reativa e proativa das informações sobre congestionamen- 


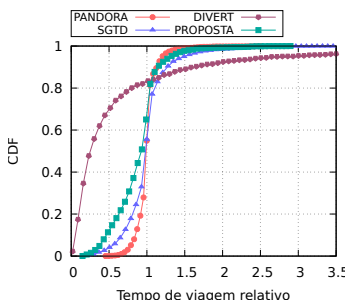

(a) Tempo de viagem relativo - trânsito normal

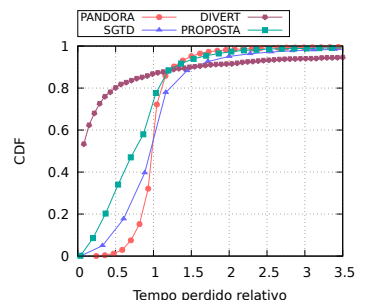

(b) Tempo perdido relativo - trânsito normal

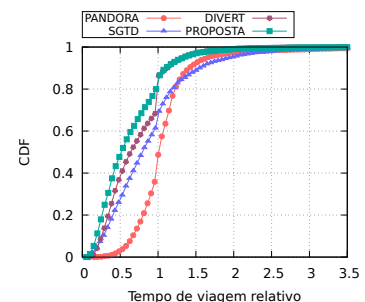

(c) Tempo de viagem relativo - fluxo intenso

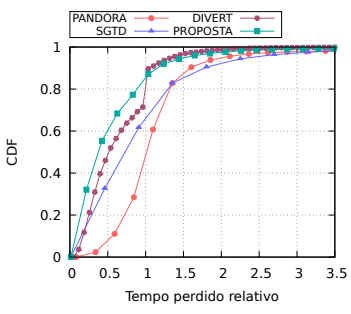

(d) Tempo perdido relativo - fluxo intenso

\section{Figura 7. Função distribuição acumulada}

tos das ruas. Além disso, a área de abrangência da disseminação proativa de informações é definida de acordo com as condições do tráfego, ou seja, em casos de tráfego intenso a área de cobertura é maior em comparação quando pequenas retenções são observadas. No caso de o veículo não possuir informações de tráfego de ruas próximas, ele executa a descoberta reativa de informação. Usando todas as informações recebidas, o veículo verifica a existência de rotas alternativas com menor congestionamento. Os resultados de simulação mostram que a solução proposta é capaz de reduzir os congestionamentos, diminuindo o tempo médio de viagem e o tempo perdido pelos veículos. A solução proposta também diminui a quantidade de mensagens para executar o gerenciamento de tráfego veicular. Como trabalhos futuros planejamos investigar algoritmos estatísticos para a descoberta de rotas alternativas com o objetivo de realizar um balanceamento de novos trajetos.

\section{Agradecimentos}

Os autores agradecem a Fundação de Amparo à Pesquisa do Estado de Minas Gerais (FAPEMIG) sob a concessão n. ${ }^{\circ}$ : APQ-03120-17, a Canadian Bureau for International Education (CBIE) através do programa The Emerging Leaders in the Americas Program (ELAP) e ao Conselho Nacional de Desenvolvimento Científico e Tecnológico (CNPq) sob a concessão n. ${ }^{\circ}: 150545 / 2018-5$. O presente trabalho foi realizado com apoio da Coordenação de Aperfeiçoamento de Pessoal de Nível Superior - Brasil (CAPES) - Código de Financiamento 001.

\section{Referências}

Ahmed, E. and Gharavi, H. (2018). Cooperative vehicular networking: A survey. IEEE Transactions on Intelligent Transportation Systems, 19(3):996-1014.

Brennand, C., Boukerche, A., Meneguette, R., and Villas, L. (2017). A novel urban traffic management mechanism based on fog. IEEE Symposium on Computers and Communications (ISCC).

Cookson, G. and Pishue, B. (2016). Inrix europe's traffic hotspots: Measuring the impact of congestion in europe. INRIX research.

de Souza, A. M., da Fonseca, N. L. S., and Villas, L. A. (2017). A fully-distributed advanced traffic management system based on opportunistic content sharing. 2017 IEEE International Conference on Communications (ICC), pages 1-6.

Ekblad, S. (1993). Stressful environments and their effects on quality ot life in third world cities. Environment and Urbanization, 5(2):125-134. 
Gomides, T., Lourenço, M., Souza, P., and Guidoni, D. (2019). Sgtd: Sistema de gerenciamento de tráfego distribuído para redes veiculares. In Anais do XXXVII Simpósio Brasileiro de Redes de Computadores e Sistemas Distribuídos, pages 1042-1055, Porto Alegre, RS, Brasil. SBC.

HCM (2010). HCM 2010 : Highway Capacity Manual. Chapter 36, Travel Time Reliability. National Research Council. Transportation Research Board.

Lopez, P. A., Behrisch, M., Bieker-Walz, L., Erdmann, J., Flötteröd, Y.-P., Hilbrich, R., Lücken, L., Rummel, J., Wagner, P., and Wießner, E. (2018). Microscopic traffic simulation using sumo. In The 21st IEEE International Conference on Intelligent Transportation Systems. IEEE.

Lourenço, M., Gomides, T. S., de Souza, F. S. H., Meneguette, R. I., and Guidoni, D. L. (2018). A traffic management service based on v2i communication for vehicular adhoc networks. In Proceedings of the 10th Latin America Networking Conference on ZZZ - LANC'18. ACM Press.

Mabogunje, A. L. (1970). Systems approach to a theory of rural-urban migration. Geographical Analysis, 2(1):1-18.

OpenStreetMap contributors (2017). Planet dump retrieved from https://planet.osm.org . https: / / www. openstreetmap.org.

Pan, J., Khan, M. A., Popa, I. S., Zeitouni, K., and Borcea, C. (2012). Proactive vehicle re-routing strategies for congestion avoidance. In 2012 IEEE 8th International Conference on Distributed Computing in Sensor Systems, pages 265-272.

Pan, J., Popa, I. S., and Borcea, C. (2017). Divert: A distributed vehicular traffic rerouting system for congestion avoidance. IEEE Transactions on Mobile Computing, 16(1):58-72.

Shahgholian, M. and Gharavian, D. (2018). Advanced traffic management systems: An overview and a development strategy. arXiv preprint arXiv:1810.02530.

Sommer, C., German, R., and Dressler, F. (2011). Bidirectionally Coupled Network and Road Traffic Simulation for Improved IVC Analysis. IEEE Transactions on Mobile Computing, 10(1):3-15.

Van Audenhove, F.-J., Korniichuk, O., Dauby, L., and Pourbaix, J. (2014). The future of urban mobility 2.0: Imperatives to shape extended mobility ecosystems of tomorrow.

Varga, A. and Hornig, R. (2008). An overview of the omnet++ simulation environment. In Proceedings of the 1st International Conference on Simulation Tools and Techniques for Communications, Networks and Systems \& Workshops, Simutools '08, pages 60:1-60:10, ICST, Brussels, Belgium, Belgium. ICST (Institute for Computer Sciences, Social-Informatics and Telecommunications Engineering).

Wang, S., Djahel, S., and McManis, J. (2014). A multi-agent based vehicles re-routing system for unexpected traffic congestion avoidance. In 17th International IEEE Conference on Intelligent Transportation Systems (ITSC), pages 2541-2548.

Wang, S., Djahel, S., and Mcmanis, J. (2015). An adaptive and vanets-based next road re-routing system for unexpected urban traffic congestion avoidance. 